\title{
Current understanding of ovarian aging
}

\author{
LI Qian ${ }^{\dagger}$, GENG XiaoDan ${ }^{\dagger}$, ZHENG Wei ${ }^{\dagger}$, TANG Jie ${ }^{\dagger}$, XU Bo \& SHI QingHua* \\ Hefei National Laboratory for Physical Sciences at Microscale and School of Life Sciences, University of Science and \\ Technology of China, Hefei 230027, China
}

Received April 23, 2012; accepted June 22, 2012

\begin{abstract}
The reproductive system of human female exhibits a much faster rate of aging than other body systems. Ovarian aging is thought to be dominated by a gradual decreasing numbers of follicles, coinciding with diminished quality of oocytes. Menopause is the final step in the process of ovarian aging. This review focuses on the mechanisms underlying the ovarian aging involving a poor complement of follicles at birth and a high rate of attrition each month, as well as the alternated endocrine factors. We also discuss the possible causative factors that contribute to ovarian aging, e.g., genetic factors, accumulation of irreparable damage of microenvironment, pathological effect and other factors. The appropriate and reliable methods to assess ovarian aging, such as quantification of follicles, endocrine measurement and genetic testing have also been discussed. Increased knowledge of the ovarian aging mechanisms may improve the prevention of premature ovarian failure.
\end{abstract}

ovarian aging, menopause, genetic factors, microenvironment, pathology, assessment

Citation: $\quad$ Li Q, Geng X D, Zheng W, et al. Current understanding of ovarian aging. Sci China Life Sci, 2012, 55: 659-669, doi: 10.1007/s11427-012-4352-5

The ovary undergoes much more serious effects of age than any other tissues of the body, and the reproductive outcome has been demonstrated to be negatively correlated with age $[1,2]$. The ovarian aging related follicle number reduction and oocyte quality decay cause the gradual decline in fertility and ultimately natural sterility. Thus, "poor ovarian reserve $(\mathrm{OR})$ " is often used synonymously with "ovarian aging".

The variability of ovarian aging among individuals is evident indicated by the large variable age at menopause. This implies that some females remain fertile until the fifth decade of life, which is physiological ovarian aging, whereas others face the loss of natural fertility in their mid-thirties, which is called premature ovarian failure (POF), and is pathological ovarian aging. The mechanisms behind gradual decreasing of the follicle pool and the decaying oocyte quality are far from being totally understood, although some progress involved in the endocrine, paracrine, metabolic and

$\dagger$ Contributed equally to this work

*Corresponding author (email: qshi@ustc.edu.cn) genetic factors has given some light to the complex puzzle.

\section{Mechanisms underlying ovarian aging}

During human fetal life, germ cells rapidly proliferate by mitosis to reach a maximum of 6-7 million oocytes by 16-20 weeks of pregnancy in human beings $[3,4]$. Formation of primordial follicles occurs in fetal ovaries by the late second trimester. Once the pool of primordial follicles formed, it serves as a source of developing follicles $[3,5,6]$, and the primordial follicles in the pool remain quiescent for many years (even for 40 years) until activated to become primary follicles [6]. Every month, a few follicles from this pool are activated and progress through the stages of primary, preantral and antral follicle. Eventually, only one follicle (dominant follicle) reaches the pre-ovulatory stage while the others undergo atresia at different developmental stages $[3,7]$. Simultaneously, the number of oocytes undergoes an inevitable decline. At birth, approximately 1-2 mil- 
lion follicles are present in ovary [8], and 300000-500000 remain by the onset of puberty $[9,10]$. During the reproductive years of life, the number of primordial follicles decreases at a steady rate of approximate 1000 follicles per month [11-13]. Actually, more than $99.9 \%$ of primordial follicles undergo atresia at different developing stages and only about 400-500 follicles reach ovulation stage in the lifetime [11].

\subsection{Follicle loss and ovary aging}

Ovarian aging is thought to be dominated by a gradual decrease in the number of primordial follicles. With ovarian aging, oocyte quality reduces with the increased incidence of miscarriages and chromosomal aberrations that usually occur after the age of 35 years [9,12,14,15] (Figure 1).

The accelerating decline of follicle pool is ascribed to a poor complement of follicles at birth and/or a high rate of attrition each month. The variation of ovarian aging among individuals is considerable. Some women show a bi-exponential decline, i.e., an abrupt decline at 38 years of age [16], while others favor an exponential decline throughout reproductive life [17]. At about 31 years old, fecundity begins gradually decreasing and the number of follicles could fall below the critical level of 25000 at approximately 37.5 years of age, indicating the beginning of the ovarian aging $[13,18]$. The decline in ovarian follicle pool dictates the onset of important reproductive processes, including decaying fecundity, natural infertility, irregular menstrual cycle and ultimately menopause [19]. The final menstrual period (menopause) occurs at an average age of 51 years [20], when only about 1000 follicles remain in the ovaries [13].

As the decrease in follicle numbers, the quality of oocyte also diminishes. The decay of oocyte quality is believed to be owing to an increase in meiotic nondisjunction, leading to an increasing rate of aneuploidy in the early embryo of older females [21-24], and also to many other factors (Figure 1). Underlying mechanisms may involve differences between germ cells when they are formed, accumulated damages in oocytes during the lifetime, or age-related decay of the quality of the granulosa cells surrounding the oocyte $[9,25]$.

A study of European women who lived between the16th and the early 19th century showed that those who married late more likely to be infertile, women who married when older than 35 years of age had twice the chance of sterility compared with those who married at 30-34 years of age [26]. Hence, delayed childbearing increases the chance of
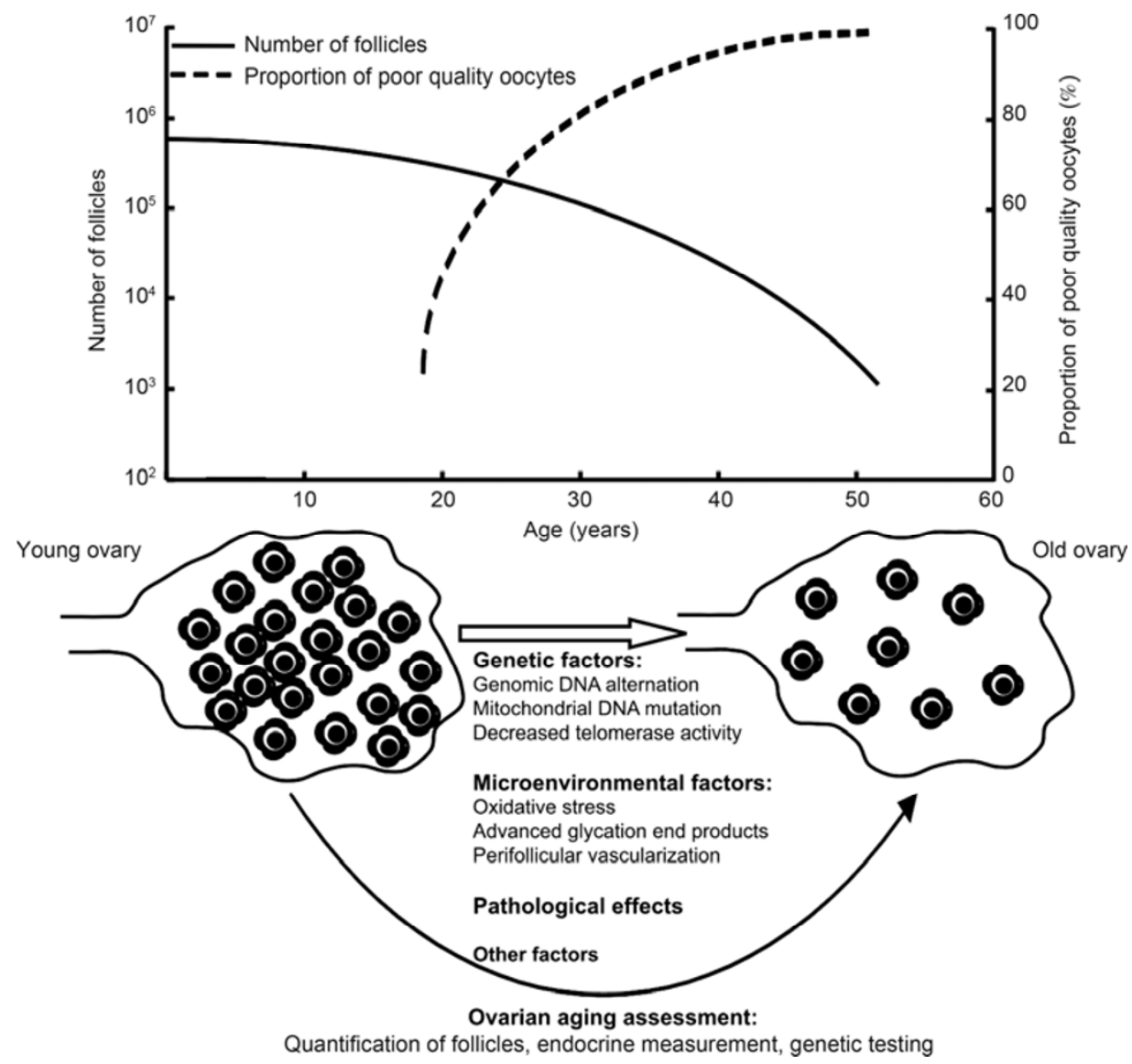

Figure 1 Schematic representation of the number of primordial follicles present in the ovaries and the quality of oocytes in relation to female age. The graph is modified from Hansen et al. [5] and de Bruin et al. [19]. 
failure of spontaneous pregnancy [26]. Delayed childbearing and reduced age-related natural sterility have resulted in an increase in the number and proportion of women more than 35 years of age who need to receive ART (assisted reproductive technologies) treatment [11]. Unfortunately, the ART treatment outcomes are also adversely influenced by advanced patient age, and to optimize treatment outcomes for these patients becomes more and more critical [27]. These indicate that the decreased quantity and quality of follicles contribute to the fertility decline.

\subsection{Follicle loss and endocrine}

Ovary, also as an important endocrinal organ, secrets sex hormones and preserves the normal menstrual cycle cooperating with hypothalamus and pituitarium. The number of the follicles in ovary has a direct relationship with the menstrual cycle during peri-menopausal stages, interacting with the levels of progesterone and gonadotropin (FSH and LH).

Epidemiologic studies in women show that hormones change at both the individual and population levels [20], and these studies extend our understanding of some key hormonal changes which occur during female reproductive aging. During the period of the transition from peri-menopausal to menopause, the number of follicles falls sharply as a result of follicle recruitment accelerating and the loss of the residual follicular stock speeding up [18], which are caused by the rising of FSH level $[11,28]$. As a result, the length of the menstrual cycle could be shortened as well as follicular phase $[3,29,30]$. The primary clinical sign of the reproductive aging is that the length of the menstrual cycle is shortened by $2-3 \mathrm{~d}[3,31]$.

The increase of FSH level is caused by declining inhibin $\mathrm{B}$ and $\mathrm{AMH}$ which are negative regulators of $\mathrm{FSH}$ level [11]. The main sources of inhibin B and AMH are the pre-antral and small antral follicles [32-34]. The depletion of the premature follicles along with aging leads to low level of inhibin B and AMH [35-40], and concurrently results in FSH accumulation [41-43]. Increasing FSH level accelerates the processes of selection and recruitment of the dominant follicles. Meanwhile, high level FSH impels granulosa cells around premature oocytes undergoing untimely maturity. The non-synchronous maturation between granulosa cells and oocytes results in more follicular atresia and loss $[11,18,44]$. As the FSH level increasing, the courses mentioned above could be magnified until the primordial follicle pool exhausted, estrogen level reduced, ultimately menopause $[45,46]$. The changes of endocrine contribute to a series of menopause symptoms caused by ovary aging and many age-related diseases, such as cardiovascular disease, osteoporosis, Alzheimer's disease, cancer, and obesity.

\section{Factors that cause ovary aging}

In general, factors affecting oocyte quality and quantity with the age are still not well defined. However, several risk factors have been indicated to be associated with premature ovarian aging and consequently the age at menopause (Figure 1), such as genetic factors, microenvironment factors and pathological factors and other factors [47].

\subsection{Genetic factors}

\subsubsection{Genomic DNA alternation}

The role of genetic factors concerning "menopausal age" has attracted increasing attention in recent years. Family history of premature menopause has been convincingly certified [48-50]. Through the menopausal age observations in sibling pairs and parent-child comparisons, it is generally accepted that genetic factors must be involved in the processes directing reproductive aging. the heritability has been estimated to range from $30 \%$ to $85 \%$ [51].

$\mathrm{POF}$ is an ovarian defect that the primordial follicle pool depletes before the age of 40 years, which can be defined as a type of ovarian aging. Therefore, POF may offer a unique model for the insights into the genetic mechanisms of ovarian aging. Several mutant mouse models such as $\mathrm{GDF9}^{-/-}$, $\mathrm{FSHR}^{-/}$and $\mathrm{ER}^{-/-}$mice have provided clues about molecules involved in the development of POF in humans [52]. Meanwhile, research in patients with POF has highlighted many candidate genes, such as GDF9, BMP15 and FOXL2, of which the micro-deletion causes early ovarian arrest [53-55]. Hamatani et al. [56] compared the oocytes between the young (5-6 weeks) and old (42-45 weeks) mice, and found that about 530 genes exhibited statistically significant differences, including genes involved in mitochondrial function (mt-Atp6, Sod1, Hspa4, Nfkbia, etc.), chromosome stability (Hook1, Tuba1, Cggbp1, etc.), oogenesis and fertilization (Madh1, Smad1, Bmpr2, etc.).

Recently, based on genome-wide linkage scanning between sibling sisters, two chromosomal regions (9q21.3 and $\mathrm{Xp} 21.3$ ) have shown suggestive linkage. No less than $28 \%$ of female fragile $\mathrm{X}$ premutation carriers may develop POF [57], and one gene in the linkage of chromosome 9 encodes a member of BCL2 family, which is participated in apoptosis $[58,59]$. Besides, a number of highly significant associations with menopausal age have been demonstrated for SNPs in regions on chromosomes 5, 6, 13, 19, and 20 by genome-wide association study in several natural menopause cohorts $[60,61]$.

In addition, the incidence of aneuploidy increases with the age. Pellestor et al. [62,63] have indicated the relationship between maternal age and chromosomal abnormalities based on the research in 1397 human oocytes obtained from IVF treatment cycles. This cytogenetic study demonstrated that chromosome non-disjunction contributed to the occurrence of aneuploidy during either meiosis I or II [64]. The occurrence of non-disjunction has been attributed to the abnormal chromosomal alignment, meiotic spindle defects 
or reduced formation of chiasmata [65-68]. Meanwhile, according to Angell et al. [69], the cohesins that hold the chromatids together during metaphase I decline gradually but constantly with age in female, which contributes to the premature separation of chromatids during meiosis and finally results in aneuploidy. In summary, both the nondisjunction and the premature separation of chromosomes contribute to aneuploidy during either meiosis I or II [70,71].

\subsubsection{Mitochondrial DNA (mtDNA) mutation}

Mitochondria play a key role in energy production, cell proliferation and apoptosis. Their own genetic material, mtDNA, is maternally inherited. mtDNA is very important, in spite of its smaller size compared with genomics DNA. Trifunovic et al. [72] engineered mutant polgA knock-in mice with a defect in the nucleus-encoded catalytic subunit of mtDNA polymerase. These mice develop a premature aging phenotype beginning at 9 months because of the accumulated mtDNA mutations. Their work provides a causative relationship between mtDNA mutations and aging phenotypes in mammals. According to the observation on luteinizing granulosa cells, Seifer et al. showed that these somatic cells contain higher levels of mtDNA deletion in the women ages $>38$ years [73], and deeply reduced antioxidant enzymes in the damage mitochondria [74], and facilitate the activation of the apoptosis in oocytes, by providing specific factors such as ceramide [75,76]. Seifer et al. [76] showed that the mtDNA 4977-bp deletion $\left(\triangle \mathrm{mtDNA}^{4977}\right.$ ) increased in the older women, which may affect the fertilization. Hamatani et al. [56] showed different expression of mitochondrial function genes that participated in the ATP metabolism and binding, as well as many other genes involved in the ubiquitin-proteasome and NF-kappa B pathway based on comparing the young and old oocyte. In abnormal mitochondria, $\left[\mathrm{Ca}^{2+}\right]$ oscillations fail to trigger ATP production which support normal physiological processes $[77,78]$, such as spindle formation, abnormal chromosomal alignment [65,79], and then affect fertility. However, these abnormalities can be rescued by micro-injecting cytoplasm from younger oocytes [80]. In short, mitochondria sustain the normal physiological functions of ovary through the ATP synthesis, calcium signaling and apoptosis.

\subsubsection{Decreased telomerase activity}

Currently, the correlation between reproduction and telomere length has received growing attractions. Telomeres are the repetitive DNA sequences at chromosomal ends, which cap the chromosomes and prevent end-to-end fusion of chromosomes $[81,82]$. The telomere length is a marker for cellular aging as it gradually shortens with successive cell division. Once telomeres reach a threshold length, cell cycle arrest, apoptosis and genomic instability will ensure $[81,83,84]$. Telomerase can maintain the telomere length and therefore ensures genomic stability [85-87]. The te- lomerase deficiency, combined with long-time exposure to ROS, may lead to the telomere shortening quickly, which will affect maternal aging on fertility [88]. Kinugawa et al. [89] indicated that the telomerase activity was present in the young ovaries, but it decreased with age. They speculated that the primordial follicle depletion was related to the decline of telomerase activity, so the telomerase maybe used as a marker of ovarian functional age. Hanna et al. [90] believed that longer telomere in the POF patients may be related to slow cell division rates or abnormal hormone exposure in these women. Butts et al. [91] considered that the aberrant telomere homeostasis was due to occult ovarian insufficiency in young women. Hamatani et al. [56] noted that the expression of TERT (telomerase reverse transcriptase) decreased during general aging and cellular senescence. In conclusion, old oocytes may be unable to maintain intact chromosomes due to the low telomerase activity, which affects the female fertility.

\subsection{Microenvironment factors in the ovarian aging}

\subsubsection{Oxidative stress (OS) and ovarian aging}

Till now, research on aging causative factors is mainly focusing on the spontaneous damages accumulated during daily biological metabolism process [92,93]. The modification of different kinds of molecules caused by oxidative stress is suggested to be one of the culprits of aging [94].

Reactive oxygen species (ROS) and reactive nitrogen species (RNS) are called free radicals and have high reactivity [95], which are generated during the biological metabolism process. The widely accepted theory is that the aging-associated cellular respiratory decline can result in increased intra-mitochondrion electron leakage and raised production of ROS, which in turn affect mtDNA stability and the function of mitochondria [96,97]. When the generation of pro-oxidants including ROS and RNS exceeds the scavenging capacity by antioxidants, the oxidative stress (OS) appears. This imbalance could cause extensive oxidative damages which then induce cytochrome $\mathrm{c}$ and other apoptosis trigger factors release from mitochondria and finally result in cell death [98-100].

Since the increase in ROS production during aging may lead to oxidized proteins accumulation, the free-SH protein level was used to explain the relationship between the intracellular oxidative stress and ovarian aging by Tatone et al. [100]. Their results clearly demonstrated a remarkable quantitative and qualitative decrease in free-SH protein in ovarian follicular fluid especially in older women. The decrease of free-SH protein with age strongly suggests that an increase of oxidative stress in the follicular microenvironment with aging [100]. Based on the same principle, Wiener-Megnazi et al. [101] reported an age-related increase in free radical activity which also correlates with a low success rate of IVF by the ROS level quantification. Thus, it is be- 
lieved that higher oxidative stress presents in follicular fluid of older women.

Long-lasting higher oxidative stress damage is considered to be involved in the process of ovarian aging [102,103]. An investigation between poor oocyte quality and oxidative stress indicated that women undergoing IVF have a significantly higher concentration of 8-OHdG (a marker of DNA oxidation degree) in the ovarian follicular fluid and a similar higher proportion of degenerative oocytes. Whereas providing those women who failed to become pregnant in the IVF-embryo transfer trial with antioxidants results in an obviously reduced intrafollicular concentration of $8-\mathrm{OHdG}$ and higher pregnant rate [104]. Recent studies have also shown that age-related decay of oocytes accompany with dysfunction of mitochondria. This adverse change arises from excessive free radicals. Mitochondrial dysfunction can cause cellular injury that increased with age [105,106]. Together with an age-related estrogen decrease, the loss of its protective effects against oxidative stress finally lead to decay of the ovary. It may account for the significantly elevated rate of congenital birth defects in the women older than 38 years [107].

Over the past few years, several studies in mammas indicated that long-term age-related oxidative stress was caused by the impairment of antioxidant enzymatic defense. Tarin et al. [108] described a sharp decrease of glutathione (GSH) and glutathione transferase (GST), both of which are involved in free radical scavenging, in ovulated mature oocytes from aged mice. It has been found that administration of antioxidants to reproductively old mice effectively rescued the adverse effects of female aging on oocyte quality [109]. Others suggested that oxidative stress level in young mouse oocytes negatively affects spindle structure by decreasing ATP production, thus give a good mimic model of the aging process [110]. Similarly, such a pattern of enzymatic defense is obviously affected by reproductive aging as oocytes from older women have a reduced ratio between CAT (catalase) and SOD (superoxide dismutase), indicating a decrease of ROS scavenging capability with aging $[111,112]$. The weakening of antioxidant defense also occurs in granulosa cells where reproductive aging accompanies with the down regulation of $\mathrm{Cu} / \mathrm{Zn} \mathrm{SOD}, \mathrm{Mn} \mathrm{SOD}$, CAT, and accumulation of oxidative damages [111].

These results indicate that oxidative stress has a negative impact on oocyte development. With the accumulation of oxidative damage through increase in ROS production and a contemporaneous lowering of antioxidant defense will finally lead to pathological ovarian aging.

\subsubsection{AGEs and ovarian aging}

The formation of AGEs (advanced glycation end products) is an irreversible process, accelerating with aging, atherosclerosis, diabetes mellitus, etc. Long-lived proteins such as collagens, nerve proteins, and lens crystallins are most vulnerable to be affected by AGEs [113]. It was suggested that
AGEs cause tissue injury either through protein crosslinking or directly binding to specific receptors termed RAGE (receptor for advanced glycation end-products) located in different cells, such as endothelium and smooth muscle cells [114]. For example, AGEs deposited in the arterial wall in Alzheimer patients may induce oxidant stress to promote further damage [115-117], as well as irreversible cross-linked proteins in vessel collagen which finally contributes to atherosclerosis [118]. Frye et al. [119] have demonstrated that the concentration of cross-links in collagen and lens proteins increased with aging in AGEs-dependent manner. The binding of AGE with their specific receptors RAGE would result in generation of intracellular oxidative stress [114]. By using different AGE inhibitors researchers found that blocking the formation of AGEs in experimental animals prevents or slows down aging [120,121]. Tatone et al. also observed that the expression and activity of enzymes for detoxifying methylglyoxal, a major precursor of AGEs, in ovaries of reproductively old mice are lower than in young mice. All the above observations indicated that the accumulation of AGEs in aging ovary and other age-related diseases is a marked event, they may account for weakened efficiency of vascularization and for the activation of oxidative response through RAGE interaction.

\subsubsection{Perifollicular vascularization and ovarian aging}

It is suggested that a healthy microenvironment is essential for follicular development and oocyte quality. An important aspect of effects is oxygen supply and paracrine regulators that are mainly supplied by perifollicular vascularization [122-124]. Indeed, modifications such as spindle and chromosome abnormalities are similar between young oocytes obtained from Graafian follicles with reduced perifollicular vascularization and old MII oocytes [108,125]. Unlike primordial and preantral follicles which gain their blood supply mainly from the stromal vessels, growing follicles depend on an ingrowth of capillaries into the theca [77]. It was well known that dominant follicles have more blood vessel than others [126,127], and oocytes deriving from follicles with complete vascularization and plenteous oxygen content $(\geqslant 3 \%)$ had higher fertilization and developmental potential [128]. Furthermore, studies of perifollicular vascularity before oocyte aspiration demonstrated a positive correlation between high-grade vascularity and improved live birth rate during IVF $[129,130]$. The hypoxia due to an inadequate ingrowth of capillaries into the theca of the mature follicle may trigger an oxidative stress. It is further supported by the observation that mitochondria of granulosa cells from aged women exhibit structural damage similar to those found in other cells with a hypoxia treatment [74,131].

In a word, the reduced oxygen content, nutritional factors, signaling molecules and others in aged ovary depending on a compromised perifollicular vascularization are typical 
characteristics of ovarian aging.

\subsection{Pathological effects}

Ovarian pathological changes may contribute to subfertility. Endometriosis is a disease that functioning endometrial tissue is outside the uterine cavity. Endometriosis is the foremost disease of reproductive-age women, $30 \%-50 \%$ of patients with endometriosis are infertility. Research has found that endometriosis was significantly related to age, peaking at ages 40-44 [132]. Laparoscopic excision or ablation lesions helps to improve pregnancy rate in patients with mild or moderate endometriosis [133]. However, excision of endometriotic ovarian cysts decreased the ovarian sensitivity to gonadotropin [134]. More evidences are needed to illustrate the effects of these coexisting diseases on ovarian function. This also reminds us that surgical exploration of infertile patients needs more cautions.

\subsection{Other factoring}

Epidemiological investigations revealed that the ovarian function would be affected by environmental, behavioral and other factors. From the 1980s onwards, a successive epidemiological survey suggested that educational level, occupation and contraceptives have effects on ovarian function and fertility [135]. Smoking is also known to advance menopause [136]. Long-term exposure to smoking has been shown to be associated with subfertility of women in later life [137]. However, the exact mechanism underlying is yet unknown. Ovarian aging is also associated with the exposure to radiation, heavy metals, pesticides and chemicals which could perturb the meiotic process and cause aneuploidy [138]. In addition, improper diet can also promote premature ovarian aging [139].

\section{Ovarian aging assessment}

In assisted reproductive technology (ART), ovarian aging has become a major detrimental factor of pregnancy achievement and maintenance. Thus, accurate assessment of ovarian aging is of utmost importance. Currently, in order to measure ovarian aging, a variety of tests have been developed (Figure 1).

\subsection{Quantification of follicles}

Ovarian aging in woman is thought to be dominated by a gradual decreasing numbers of follicles, coinciding with diminished quality of the oocytes within the follicles [9]. Total ovarian reserve (TOR) contains primordial follicles (not growing follicles, NGFs) and early growing follicles (GFs), thus the assessment of TOR could reflect the ovarian condition and predict menopausal age.
Theoretically, the most direct representation of the ovarian reserve (OR) is the quantity of ovarian NGFs. The number of primordial follicles in the peri-menopausal ovaries are only about $1 / 10$ of that in the ovaries with the regular menstrual cycles, and primordial follicles are hardly found in postmenopausal ovaries [140]. Ovarian biopsy from 60 infertile women aged 19-45 years showed a significant negative correlation between age and follicular density and ovarian volume [141]. However, the follicle density within the cortex has been shown to vary greatly in the same ovary and the minimum number of follicles required for fertility are also unknown [107,142], invasive ovarian biopsy was concluded not to be a reliable test of OR.

Currently, noninvasive and available ultrasound tests of ovarian aging include AFC (antral follicle count), ovarian volume and ovarian blood flow. Some reports showed that AFC was found to have a better reliability than ovarian volume to predict ovarian response during ART [143]. In recent years, AFC has been demonstrated to be correlated with the occurrence of the menopausal transition [144], and to be related strongly with the quantitative aspects of OR. Considering the ease of measurement, low cost and reliability, AFC has become one of the most commonly used ultrasonic markers of ovarian reserve in clinical practice [145]. In woman with poor ovarian reserve, stromal blood flow has been shown to be reduced or absent by Doppler assessment [146] . It is possible that ovarian stromal flow is used in the prediction of premature ovarian aging.

\subsection{Endocrine measurement}

Ovarian is an endocrine organ and functions through the feedback of gonadal hormones and gonadotropins. The decreased size of AFC is the main cause of the altered feedback, with a reduction in inhibin $\mathrm{B}$ secretion in the initial stages and estradiol and inhibin A output in later stages. This serological testing method is convenient and with high reproducibility.

During the early follicular phase, basal hormone tests including serum follicle-stimulating hormone (FSH), E2 and FSH/luteinizing hormone (LH) ratio, have been traditionally employed for OR assessment. The elevated FSH level is an irrefutable sign of ovarian aging, which only rise 10 years before the menopause or when women are becoming infertile. Therefore, the change of FSH level is only a short-term predictor $[147,148]$. The levels of estradiol and inhibin A remain stable in early follicular phase in women, until the later phase of the menopausal transition $[149,150]$. Thus, the predictive ability of these tests to ovarian aging is limited in some extents.

Anti-mullerian hormone (AMH) and inhibin are the TGF-B (transforming growth factor-B) superfamily members. AMH is secreted into serum preferentially by small antral follicles $[151,152]$. Previous studies have shown that the $\mathrm{AMH}$ level is strongly related to inhibin $\mathrm{B}, \mathrm{FSH}$ and 
AFC in IVF $[153,154]$. With the decrease in the number of $\mathrm{AFC}$, the AMH serum level diminishes as well [144,155-157]. In contrast to other markers, one of the main advantages of AMH is the stability of its serum level during the entire menstrual cycle [158]. Therefore, in clinical practice, $\mathrm{AMH}$ seems to be a suitable marker for OR prediction [159]. Nevertheless, serum AMH levels would invariably become undetectable near menopause [160] and this low-value makes it difficult to predict menopause age accurately.

Inhibin B is primarily produced by antral follicles which are FSH-sensitive [161,162]. A reduction in cohort size with aging is accompanied by elevated FSH levels and decreased inhibin B secretion [163], however, inhibin B is often viewed as a rather late marker of decreased follicle numbers $[43,163]$.

\subsection{Genetic testing}

Increasing attention has been paid to the genetic factors of ovarian aging and natural menopausal age. The high heritability for the age of menopause indicates that there is a genetic effect in ovarian aging [9]. Genes whose alteration causes POF have also been used for the assessment of ovarian aging. These genes are those that associated with primordial follicle formation and growth, such as FIGLA, BMP15 and GDF9 gene [164,165], and those involved in hormone production, such as FSHR, LHR, FSH, CYP19 and CYP17 [164,165]. As the substantial advances in genome research and its applications to the field of reproduction, genetic diagnosis of ovarian aging will become more popular.

\section{Perspective}

The mechanism underlying ovarian aging is exceptionally complex. It has been known that the OR is decreasing over time, but how this reserve is established in fetal ovaries, and whether the germline stem cells exist, are still unknown. With the continuous development of science and technology, transgenic/knockout technology and gene chips could be used to study the intrinsic molecular mechanisms of ovarian aging. Proper assessment of ovarian aging is of utmost importance. The diagnosis of predictability at appropriate timing may guide pregnancy achievement or fertility preservation in women at risk.

This work was supported by the National Basic Research Program of China (Grant Nos. 2012CB944402, 2011CB944501 and 2007CB947401) and Program of Knowledge Innovation of Chinese Academy of Sciences (Grant No. KSCX2-EW-R-07).

1 Templeton A, Morris J K, Parslow W. Factors that affect outcome of in vitro fertilisation treatment. Lancet, 1996, 348: 1402-1406
2 Baird D T, Collins J, Egozcue J, et al. Fertility and ageing. Hum Reprod Update, 2005, 11: 261-276

3 Broekmans F J, Knauff E A, te Velde E R, et al. Female reproductive ageing: Current knowledge and future trends. Trends Endocrinol Metab, 2007, 18: 58-65

4 Djahanbakhch O, Ezzati M, Zosmer A. Reproductive ageing in women. J Pathol, 2007, 211: 219-231

5 Hansen K R, Knowlton N S, Thyer A C, et al. A new model of reproductive aging: The decline in ovarian non-growing follicle number from birth to menopause. Hum Reprod, 2008, 23: 699-708

6 McGee E A, Hsueh A J. Initial and cyclic recruitment of ovarian follicles. Endocr Rev, 2000, 21: 200-214

7 Hirshfield A N. Development of follicles in the mammalian ovary. Int Rev Cytol, 1991, 124: 43-101

8 Markstrom E, Svensson E, Shao R, et al. Survival factors regulating ovarian apoptosis - dependence on follicle differentiation. Reproduction, 2002, 123: 23-30

9 te Velde E R, Pearson P L. The variability of female reproductive ageing. Hum Reprod Update, 2002, 8: 141-154

10 Block E. A quantitative morphological investigation of the follicular system in newborn female infants. Acta anatomica, 1953, 17: 201-206

11 Alviggi C, Humaidan P, Howles C M, et al. Biological versus chronological ovarian age: Implications for assisted reproductive technology. Reprod Biol Endocrinol, 2009, 7: 101

12 te Velde E R, Scheffer G J, Dorland M, et al. Developmental and endocrine aspects of normal ovarian aging. Mol Cell Endocrinol, 1998, 145: 67-73

13 Faddy M J, Gosden R G, Gougeon A, et al. Accelerated disappearance of ovarian follicles in mid-life: Implications for forecasting menopause. Hum Reprod, 1992, 7: 1342-1346

14 Klein N A, Soules M R. Endocrine changes of the perimenopause. Clin Obstet Gynecol, 1998, 41: 912-920

15 Navot D, Bergh P A, Williams M A, et al. Poor oocyte quality rather than implantation failure as a cause of age-related decline in female fertility. Lancet, 1991, 337: 1375-1377

16 Faddy M J, Gosden R G. A mathematical model of follicle dynamics in the human ovary. Hum Reprod, 1995, 10: 770-775

17 Faddy M J. Follicle dynamics during ovarian ageing. Mol Cell Endocrinol, 2000, 163: 43-48

18 Nikolaou D, Templeton A. Early ovarian ageing: A hypothesis. Detection and clinical relevance. Hum Reprod, 2003, 18: 11371139

19 de Bruin J P, Dorland M, Spek E R, et al. Age-related changes in the ultrastructure of the resting follicle pool in human ovaries. Biol Reprod, 2004, 70: 419-424

20 Treloar A E. Menstrual cyclicity and the pre-menopause. Maturitas, 1981, 3: 249-264

21 Battaglia D E, Goodwin P, Klein N A, et al. Influence of maternal age on meiotic spindle assembly in oocytes from naturally cycling women. Hum Reprod, 1996, 11: 2217-2222

22 Kuliev A, Cieslak J, Verlinsky Y. Frequency and distribution of chromosome abnormalities in human oocytes. Cytogenet Genome Res, 2005, 111: 193-198

23 Hunt P A, Hassold T J. Human female meiosis: What makes a good egg go bad? Trends Genet, 2008, 24: 86-93

24 Pellestor F, Anahory T, Hamamah S. Effect of maternal age on the frequency of cytogenetic abnormalities in human oocytes. Cytogenet Genome Res, 2005, 111: 206-212

25 Warburton D. Biological aging and the etiology of aneuploidy. Cytogenet Genome Res, 2005, 111: 266-272

26 Menken J, Trussell J, Larsen U. Age and infertility. Science, 1986, 233: 1389-1394

27 Howles C M, Kim C H, Elder K. Treatment strategies in assisted reproduction for women of advanced maternal age. Int Surgery, 2006, 91: S37-54

28 Gougeon A, Ecochard R, Thalabard J C. Age-related changes of the 
population of human ovarian follicles: Increase in the disappearance rate of non-growing and early-growing follicles in aging women. Biol Reprod, 1994, 50: 653-663

29 van Zonneveld P, Scheffer G J, Broekmans F J M, et al. Do cycle disturbances explain the age-related decline of female fertility? Cycle characteristics of women aged over 40 years compared with a reference population of young women. Hum Reprod, 2003, 18: 495-501

30 Santoro N, Isaac B, Neal-Perry G, et al. Impaired folliculogenesis and ovulation in older reproductive aged women. J Clin Endocrinol Metab, 2003, 88: 5502-5509

31 Treloar A E, Boynton R E, Behn B G, et al. Variation of the human menstrual cycle through reproductive life. Int J Fertil, 1967, 12: 77-126

32 Yamoto M, Minami S, Nakano R, et al. Immunohistochemical localization of inhibin/activin subunits in human ovarian follicles during the menstrual cycle. J Clin Endocrinol Metab, 1992, 74: 989-993

33 Schwall R H, Mason A J, Wilcox J N, et al. Localization of inhibin/activin subunit mrnas within the primate ovary. Mol Endocrinol, 1990, 4: 75-79

34 Roberts V J, Barth S, el-Roeiy A, et al. Expression of inhibin/activin subunits and follistatin messenger ribonucleic acids and proteins in ovarian follicles and the corpus luteum during the human menstrual cycle. J Clin Endocrinol Metab, 1993, 77: 1402-1410

35 Klein N A, Illingworth P J, Groome N P, et al. Decreased inhibin B secretion is associated with the monotropic FSH rise in older, ovulatory women: A study of serum and follicular fluid levels of dimeric inhibin $\mathrm{a}$ and $\mathrm{b}$ in spontaneous menstrual cycles. J Clin Endocr Metab, 1996, 81: 2742-2745

36 Klein N A, Houmard B S, Hansen K R, et al. Age-related analysis of inhibin a, inhibin $\mathrm{B}$, and activin a relative to the intercycle monotropic follicle-stimulating hormone rise in normal ovulatory women. J Clin Endocrinol Metab, 2004, 89: 2977-2981

37 Danforth D R, Arbogast L K, Mroueh J, et al. Dimeric inhibin: A direct marker of ovarian aging. Fertil Steril, 1998, 70: 119-123

38 Hurwitz J M, Santoro N. Inhibins, activins, and follistatin in the aging female and male. Semin Reprod Med, 2004, 22: 209-217

39 Santoro N, Adel T, Skurnick J H. Decreased inhibin tone and increased activin a secretion characterize reproductive aging in women. Fertil Steril, 1999, 71: 658-662

40 Reame N E, Wyman T L, Phillips D J, et al. Net increase in stimulatory input resulting from a decrease in inhibin B and an increase in activin a may contribute in part to the rise in follicular phase follicle-stimulating hormone of aging cycling women. J Clin Endocrinol Metab, 1998, 83: 3302-3307

41 Randolph J F Jr., Sowers M, Bondarenko I V, et al. Change in estradiol and follicle-stimulating hormone across the early menopausal transition: Effects of ethnicity and age. $\mathrm{J}$ Clin Endocrinol Metab, 2004, 89: 1555-1561

42 Santoro N. The menopausal transition. Am J Med, 2005, 118: 813

43 Burger H G, Hale G E, Robertson D M, et al. A review of hormonal changes during the menopausal transition: Focus on findings from the melbourne women's midlife health project. Hum Reprod Update, 2007, 13: 559-565

44 Beemsterboer S N, Homburg R, Gorter N A, et al. The paradox of declining fertility but increasing twinning rates with advancing maternal age. Hum Reprod, 2006, 21: 1531-1532

45 Gleicher N, Weghofer A, Barad D H. Defining ovarian reserve to better understand ovarian aging. Reprod Biol Endocrinol, 2011, 9: 23

46 Ferrell R J, Sowers M. Longitudinal, epidemiologic studies of female reproductive aging. Ann N Y Acad Sci, 2010, 1204: 188197

47 Younis J S. Ovarian aging: Latest thoughts on assessment and management. Curr Opin Obstet Gynecol, 2011, 23: 427-434

48 Murabito J M, Yang Q, Fox C, et al. Heritability of age at natural menopause in the framingham heart study. J Clin Endocrinol Metab,
2005, 90: 3427-3430

49 Torgerson D J, Thomas R E, Reid D M. Mothers and daughters menopausal ages: Is there a link? Eur J Obstet Gynecol Reprod Biol, 1997, 74: 63-66

50 van Asselt K M, Kok H S, Pearson P L, et al. Heritability of menopausal age in mothers and daughters. Fertil Steril, 2004, 82: $1348-1351$

51 Wicks J, Treloar S A, Martin N G. Using identity-by-de scent information in affected sib pairs to increase the efficiency of genetic association studies. Twin Res, 2004, 7: 211-216

52 Jagarlamudi K, Reddy P, Adhikari D, et al. Genetically modified mouse models for premature ovarian failure (POF). Mol Cell Endocrinol, 2010, 315: 1-10

53 Altshuler D, Brooks L D, Chakravarti A, et al. A haplotype map of the human genome. Nature, 2005, 437: 1299-1320

54 Lakhal B, Laissue P, Braham R, et al. Bmp15 and premature ovarian failure: Causal mutations, variants, polymorphisms? Clin Endocrinol, 2010, 72: 425-426

55 Willer C J, Scott L J, Bonnycastle L L, et al. Tag snp selection for finnish individuals based on the ceph utah hapmap database. Genet Epidemiol, 2006, 30: 180-190

56 Hamatani T, Falco G, Carter M G, et al. Age-associated alteration of gene expression patterns in mouse oocytes. Hum Mol Genet, 2004, 13: 2263-2278

57 Welt C K, Smith P C, Taylor A E. Evidence of early ovarian aging in fragile X premutation carriers. J Clin Endocr Metab, 2004, 89: 4569-4574

58 Hsu S Y, Hsueh A J W. Tissue-specific Bcl-2 protein partners in apoptosis: An ovarian paradigm. Physiol Rev, 2000, 80: 593-614

59 Petros A M, Olejniczak E T, Fesik S W. Structural biology of the Bcl-2 family of proteins. Bba-Mol Cell Res, 2004, 1644: 83-94

60 He C Y, Kraft P, Chen C, et al. Genome-wide association studies identify loci associated with age at menarche and age at natural menopause. Nat Genet, 2009, 41: 724-728

61 Stolk L, Zhai G, van Meurs J B J, et al. Loci at chromosomes 13, 19 and 20 influence age at natural menopause. Nat Genet, 2009, 41: 645-647

62 Pellestor F, Anahory T, Hamamah S. Effect of maternal age on the frequency of cytogenetic abnormalities in human oocytes. Cytogenet Genome Res, 2005, 111: 206-212

63 Pellestor F, Andreo B, Anahory T, et al. The occurrence of aneuploidy in human: Lessons from the cytogenetic studies of human oocytes. European J Med Genet, 2006, 49: 103-116

64 Colbere-Garapin F, Duncan G, Pavio N, et al. An approach to understanding the mechanisms of poliovirus persistence in infected cells of neural or non-neural origin. Clin Diagn Virol, 1998, 9: 107-113

65 Battaglia D E, Goodwin P, Klein N A, et al. Influence of maternal age on meiotic spindle assembly in oocytes from naturally cycling women. Hum Reprod, 1996, 11: 2217-2222

66 Ghosh S, Bhaumik P, Ghosh P, et al. Chromosome 21 non-disjunction and down syndrome birth in an indian cohort: Analysis of incidence and aetiology from family linkage data. Genet Res, 2010, 92: 189-197

67 Hassold T, Abruzzo M, Adkins K, et al. Human aneuploidy: Incidence, origin, and etiology. Environ Mol Mutag, 1996, 28: $167-175$

68 Macdonald M, Hassold T, Harvey J, et al. The origin of 47,XXY and 47,XXX aneuploidy-heterogeneous mechanisms and role of aberrant recombination. Hum Mol Genet, 1994, 3: 1365-1371

69 Angell R R. Predivision in human oocytes at meiosis- $\mathrm{I}-\mathrm{a}$ mechanism for trisomy formation in man. Hum Genet, 1991, 86: 383-387

70 Petersen M B. Origin and mechanisms of nondisjunction in human autosomal trisomies. Cytogenet Cell Genet, 1999, 85: 21-21

71 Watanabe Y, Nurse P. Cohesin rec8 is required for reductional chromosome segregation at meiosis. Nature, 1999, 400: 461-464

72 Trifunovic A, Wredenberg A, Falkenberg M, et al. Premature ageing 
in mice expressing defective mitochondrial DNA polymerase. Nature, 2004, 429: 417-423

73 Seifer D B, DeJesus V, Hubbard K. Mitochondrial deletions in luteinized granulosa cells as a function of age in women undergoing in vitro fertilization. Fertil Steril, 2002, 78: 1046-1048

74 Tatone C, Carbone M C, Falone S, et al. Age-dependent changes in the expression of superoxide dismutases and catalase are associated with ultrastructural modifications in human granulosa cells. Mol Hum Reprod, 2006, 12: 655-660

75 Perez G I, Jurisicova A, Matikainen T, et al. A central role for ceramide in the age-related acceleration of apoptosis in the female germline. FASEB J, 2005, 19: 860-862

76 Perez G I, Tilly J L. Cumulus cells are required for the increased apoptotic potential in oocytes of aged mice. Hum Reprod, 1997, 12: 2781-2783

77 Gaulden M E. Maternal age effect: The enigma of down syndrome and other trisomic conditions. Mutat Res, 1992, 296: 69-88

78 Gordo A C, Rodrigues P, Kurokawa M, et al. Intracellular calcium oscillations signal apoptosis rather than activation in in vitro aged mouse eggs. Biol Reprod, 2002, 66: 1828-1837

79 Eichenlaub-Ritter U, Vogt E, Yin H, et al. Spindles, mitochondria and redox potential in ageing oocytes. Reprod Biomed Online, 2004, 8: $45-58$

80 Klein J, Sauer M V. Assessing fertility in women of advanced reproductive age. Am J Obstet Gynecol, 2001, 185: 758-770

81 Blasco M A, Gasser S M, Lingner J. Telomeres and telomerase. Genes Dev, 1999, 13: 2353-2359

82 Greider C W. Telomeres, telomerase and senescence. Bioessays, 1990, 12: 363-369

83 Harley C B, Futcher A B, Greider C W. Telomeres shorten during aging of human fibroblasts. Nature, 1990, 345: 458-460

84 Vonzglinicki T, Saretzki G, Docke W, et al. Mild hyperoxia shortens telomeres and inhibits proliferation of fibroblasts - a model for senescence. Exp Cell Res, 1995, 220: 186-193

85 Collins K. Structure and function of telomerase. Curr Opin Cell Biol, 1996, 8: 374-380

86 Harrington L A, Greider C W. Telomerase primer specificity and chromosome healing. Nature, 1991, 353: 451-454

87 Yu G L, Blackburn E H. Developmentally programmed healing of chromosomes by telomerase in tetrahymena. Cell, 1991, 67: 823832

88 Keefe D L, Marquard K, Liu L. The telomere theory of reproductive senescence in women. Curr Opin Obstet Gyn, 2006, 18: 280-285

89 Kinugawa C, Murakami T, Okamura K, et al. Telomerase activity in normal ovaries and premature ovarian failure. Tohoku J Exp Med, 2000, 190: 231-238

90 Hanna C W, Bretherick K L, Gair J L, et al. Telomere length and reproductive aging. Hum Reprod, 2009, 24: 1206-1211

91 Butts S, Riethman H, Ratcliffe S, et al. Correlation of telomere length and telomerase activity with occult ovarian insufficiency. J Clin Endocrinol Metab, 2009, 94: 4835-4843

92 Moore G E. Biochemical and cellular mechanisms of aging and degenerative disease: Excessive, poor-quality caloric intake may deplete essential nutrients and interfere with cellular processes to produce degenerative damage. Med Hypotheses, 2008, 70: 768775

93 Yin D, Chen K. The essential mechanisms of aging: Irreparable damage accumulation of biochemical side-reactions. Exp Gerontol, 2005, 40: $455-465$

94 Sohal R S. Role of oxidative stress and protein oxidation in the aging process. Free Radic Biol Med, 2002, 33: 37-44

95 Agarwal A, Aponte-Mellado A, Premkumar B J, et al. The effects of oxidative stress on female reproduction: A review. Reprod Biol Endocrinol, 2012, 10: 49

96 Sahin E, Depinho R A. Axis of ageing: Telomeres, p53 and mitochondria. Nat Rev Mol Cell Biol, 2012, 13: 397-404

97 Miquel J, Economos A C, Fleming J, et al. Mitochondrial role in cell aging. Exp Gerontol, 1980, 15: 575-591
98 Orrenius S, Gogvadze V, Zhivotovsky B. Mitochondrial oxidative stress: Implications for cell death. Ann Rev Pharmacol Toxicol, 2007, 47: 143-183

99 Vercesi A E, Kowaltowski A J, Oliveira H C, et al. Mitochondrial $\mathrm{Ca}^{2+}$ transport, permeability transition and oxidative stress in cell death: Implications in cardiotoxicity, neurodegeneration and dyslipidemias. Front Biosci, 2006, 11: 2554-2564

100 Tatone C, Amicarelli F, Carbone M C, et al. Cellular and molecular aspects of ovarian follicle ageing. Hum Reprod Update, 2008, 14: $131-142$

101 Wiener-Megnazi Z, Vardi L, Lissak A, et al. Oxidative stress indices in follicular fluid as measured by the thermochemiluminescence assay correlate with outcome parameters in in vitro fertilization. Fertil Steril, 2004, 82: 1171-1176

102 Tarin J J. Aetiology of age-associated aneuploidy: A mechanism based on the 'free radical theory of ageing'. Hum Reprod, 1995, 10: $1563-1565$

103 Tarin J J. Potential effects of age-associated oxidative stress on mammalian oocytes/embryos. Mol Hum Reprod, 1996, 2: 717724

104 Tamura H, Takasaki A, Miwa I, et al. Oxidative stress impairs oocyte quality and melatonin protects oocytes from free radical damage and improves fertilization rate. J Pineal Res, 2008, 44: 280-287

105 Liu L, Keefe D L. Cytoplasm mediates both development and oxidation-induced apoptotic cell death in mouse zygotes. Biol Reprod, 2000, 62: 1828-1834

106 Liu L, Trimarchi J R, Keefe D L. Involvement of mitochondria in oxidative stress-induced cell death in mouse zygotes. Biol Reprod, 2000, 62: 1745-1753

107 Lass A, Skull J, McVeigh E, et al. Measurement of ovarian volume by transvaginal sonography before ovulation induction with human menopausal gonadotrophin for in-vitro fertilization can predict poor response. Hum Reprod, 1997, 12: 294-297

108 Van Blerkom J. The influence of intrinsic and extrinsic factors on the developmental potential and chromosomal normality of the human oocyte. J Soc Gynecol Investig, 1996, 3: 3-11

109 Tarin J J, Perez-Albala S, Cano A. Oral antioxidants counteract the negative effects of female aging on oocyte quantity and quality in the mouse. Mol Reprod Dev, 2002, 61: 385-397

110 Zhang X, Wu X Q, Lu S, et al. Deficit of mitochondria-derived ATP during oxidative stress impairs mouse MII oocyte spindles. Cell Res, 2006, 16: 841-850

111 Carbone M C, Tatone C, Delle Monache S, et al. Antioxidant enzymatic defences in human follicular fluid: Characterization and age-dependent changes. Mol Hum Reprod, 2003, 9: 639-643

112 Gonzalez-Parraga P, Hernandez J A, Arguelles J C. Role of antioxidant enzymatic defences against oxidative stress $\mathrm{H}_{2} \mathrm{O}_{2}$ and the acquisition of oxidative tolerance in candida albicans. Yeast, 2003, 20: 1161-1169

113 Yim M B, Yim H S, Lee C, et al. Protein glycation: Creation of catalytic sites for free radical generation. Ann N Y Acad Sci, 2001, 928: 48-53

114 Schmidt A M, Yan S D, Yan S F, et al. The biology of the receptor for advanced glycation end products and its ligands. Biochim Biophys Acta, 2000, 1498: 99-111

115 Mullarkey C J, Edelstein D, Brownlee M. Free radical generation by early glycation products: A mechanism for accelerated atherogenesis in diabetes. Biochem Biophys Res Commun, 1990, 173: 932-939

116 Sakurai T, Tsuchiya S. Superoxide production from nonenzymatically glycated protein. FEBS Lett, 1988, 236: 406-410

117 Wen Y, Skidmore J C, Porter-Turner M M, et al. Relationship of glycation, antioxidant status and oxidative stress to vascular endothelial damage in diabetes. Diabetes Obes Metab, 2002, 4: 305-308

118 Soldatos G, Cooper M E. Advanced glycation end products and vascular structure and function. Curr Hypertens Rep, 2006, 8: $472-478$ 
119 Frye E B, Degenhardt T P, Thorpe S R, et al. Role of the maillard reaction in aging of tissue proteins. Advanced glycation end product-dependent increase in imidazolium cross-links in human lens proteins. J Biol Chem, 1998, 273: 18714-18719

120 Kim J, Kim O S, Kim C S, et al. Accumulation of argpyrimidine, a methylglyoxal-derived advanced glycation end product, increases apoptosis of lens epithelial cells both in vitro and in vivo. Exp Mol Med, 2012, 44: 167-175

121 Mizutani K, Ikeda K, Tsuda K, et al. Inhibitor for advanced glycation end products formation attenuates hypertension and oxidative damage in genetic hypertensive rats. J Hypertens, 2002, 20: 1607-1614

122 Dixit H, Rao L K, Padmalatha V V, et al. Missense mutations in the BMP15 gene are associated with ovarian failure. Hum Genet, 2006, 119: 408-415

123 Laissue P, Christin-Maitre S, Touraine $\mathrm{P}$, et al. Mutations and sequence variants in GDF9 and BMP15 in patients with premature ovarian failure. Eur J Endocrinol, 2006, 154: 739-744

124 Schmidt D, Ovitt C E, Anlag K, et al. The murine winged-helix transcription factor Fox12 is required for granulosa cell differentiation and ovary maintenance. Development, 2004, 131: 933-942

125 Van Blerkom J, Antczak M, Schrader R. The developmental potential of the human oocyte is related to the dissolved oxygen content of follicular fluid: Association with vascular endothelial growth factor levels and perifollicular blood flow characteristics. Hum Reprod, 1997, 12: 1047-1055

126 Modlich U, Kaup F J, Augustin H G. Cyclic angiogenesis and blood vessel regression in the ovary: Blood vessel regression during luteolysis involves endothelial cell detachment and vessel occlusion. Lab Invest, 1996, 74: 771-780

127 Redmer D A, Reynolds L P. Angiogenesis in the ovary. Rev Reprod, 1996, 1: 182-192

128 Huey S, Abuhamad A, Barroso G, et al. Perifollicular blood flow doppler indices, but not follicular po2, pco2, or ph, predict oocyte developmental competence in in vitro fertilization. Fertil Steril, 1999, 72: 707-712

129 Bhal P S, Pugh N D, Gregory L, et al. Perifollicular vascularity as a potential variable affecting outcome in stimulated intrauterine insemination treatment cycles: A study using transvaginal power doppler. Hum Reprod, 2001, 16: 1682-1689

130 Borini A, Maccolini A, Tallarini A, et al. Perifollicular vascularity and its relationship with oocyte maturity and IVF outcome. Ann N Y Acad Sci, 2001, 943: 64-67

131 Amicarelli F, Ragnelli A M, Aimola P, et al. Age-dependent ultrastructural alterations and biochemical response of rat skeletal muscle after hypoxic or hyperoxic treatments. Biochim Biophys Acta, 1999, 1453: 105-114

132 Vessey M P, Villard-Mackintosh L, Painter R. Epidemiology of endometriosis in women attending family planning clinics. BMJ, 1993, 306: 182-184

133 Allaire C. Endometriosis and infertility: A review. J Reprod Med, 2006, 51: 164-168

134 Jacobson T Z, Duffy J M, Barlow D, et al. Laparoscopic surgery for subfertility associated with endometriosis. Cochrane Database Syst Rev, 2010, CD001398

135 Islam M N, Islam M M. Biological and behavioural determinants of fertility in bangladesh: 1975-1989. Asia Pac Popul J, 1993, 8: 3-18

136 McKinlay S M, Brambilla D J, Posner J G. The normal menopause transition. Maturitas, 2008, 61: 4-16

137 Jensen T K, Henriksen T B, Hjollund N H, et al. Adult and prenatal exposures to tobacco smoke as risk indicators of fertility among 430 danish couples. Am J Epidemiol, 1998, 148: 992-997

138 Sharara F I, Scott R T Jr., Seifer D B. The detection of diminished ovarian reserve in infertile women. Am J Obstet Gynecol, 1998, 179: 804-812

139 Goldin A, Beckman J A, Schmidt A M, et al. Advanced glycation end products: Sparking the development of diabetic vascular injury. Circulation, 2006, 114: 597-605

140 Richardson S J, Nelson J F. Follicular depletion during the menopausal transition. Ann N Y Acad Sci, 1990, 592: 13-20; discussion 44-51

141 Lass A, Silye R, Abrams D C, et al. Follicular density in ovarian biopsy of infertile women: A novel method to assess ovarian reserve. Hum Reprod, 1997, 12: 1028-1031

142 Lambalk C B, de Koning C H, Flett A, et al. Assessment of ovarian reserve. Ovarian biopsy is not a valid method for the prediction of ovarian reserve. Hum Reprod, 2004, 19: 1055-1059

143 Hendriks D J, Kwee J, Mol B W J, et al. Ultrasonography as a tool for the prediction of outcome in ivf patients: A comparative meta-analysis of ovarian volume and antral follicle count. Fertil Steril, 2007, 87: 764-775

144 van Rooij I A J, Tonkelaar I, Broekmans F J M, et al. Anti-mullerian hormone is a promising predictor for the occurrence of the menopausal transition. Menopause, 2004, 11: 601

145 Broekmans F J, de Ziegler D, Howles C M, et al. The antral follicle count: Practical recommendations for better standardization. Fertil Steril, 2010, 94: 1044-1051

146 Younis J S, Haddad S, Matilsky M, et al. Undetectable basal ovarian stromal blood flow in infertile women is related to low ovarian reserve. Gynecol Endocrinol, 2007, 23: 284-289

147 Robertson D M. Anti-müllerian hormone as a marker of ovarian reserve: An update. Women's Health, 2008, 4: 137-141

148 Sowers M F R, Eyvazzadeh A D, McConnell D, et al. Anti-mullerian hormone and inhibin $\mathrm{B}$ in the definition of ovarian aging and the menopause transition. J Clin Endocrinol Metab, 2008, 93: 3478-3483

149 Sharov A A, Falco G, Piao Y, et al. Effects of aging and calorie restriction on the global gene expression profiles of mouse testis and ovary. BMC Biol, 2008, 6: 24

150 Welt C K, Smith Z A, Pauler D K, et al. Differential regulation of inhibin a and inhibin B by luteinizing hormone, follicle-stimulating hormone, and stage of follicle development. J Clin Endocr Metab, 2001, 86: 2531-2537

151 Catteau-Jonard S, Pigny P, Reyss A C, et al. Changes in serum anti-müllerian hormone level during low-dose recombinant follicular-stimulating hormone therapy for anovulation in polycystic ovary syndrome. J Clin Endocr Metab, 2007, 92: 4138-4143

152 Fanchin R, Schonauer L M, Righini C, et al. Serum anti-mullerian hormone is more strongly related to ovarian follicular status than serum inhibin B, estradiol, FSH and LH on day 3. Hum Reprod, 2003, 18: 323-327

153 Ficicioglu C, Kutlu T, Baglam E, et al. Early follicular antimullerian hormone as an indicator of ovarian reserve. Fertil Steril, 2006, 85: 592-596

154 Visser J A, de Jong F H, Laven J S E, et al. Anti-müllerian hormone: A new marker for ovarian function. Reproduction, 2006, 131: 1-9

155 de Vet A, Laven J S E, de Jong F H, et al. Antimüllerian hormone serum levels: A putative marker for ovarian aging. Fertil Steril, 2002, 77: 357-362

156 Hagstad A, Johansson S, Wilhelmsson C, et al. Gynaecology of middle-aged women - menstrual and reproductive histories. Maturitas, 1985, 7: 99-113

157 van Rooij I A J, Broekmans F J M, Scheffer G J, et al. Serum antimüllerian hormone levels best reflect the reproductive decline with age in normal women with proven fertility: A longitudinal study. Fertil Steril, 2005, 83: 979-987

158 Fanchin R, Taieb J, Lozano D H M, et al. High reproducibility of serum anti-müllerian hormone measurements suggests a multi-staged follicular secretion and strengthens its role in the assessment of ovarian follicular status. Hum Reprod, 2005, 20: 923-927

159 La Marca A, Sighinolfi G, Radi D, et al. Anti-müllerian hormone $(\mathrm{AMH})$ as a predictive marker in assisted reproductive technology (ART). Hum Reprod Update, 2010, 16: 113-130 
160 Sowers M R, Eyvazzadeh A D, McConnell D, et al. Anti-mullerian hormone and inhibin $\mathrm{B}$ in the definition of ovarian aging and the menopause transition. J Clin Endocrinol Metab, 2008, 93: 3478-3483

161 Groome N P, Illingworth P J, O’Brien M, et al. Detection of dimeric inhibin throughout the human menstrual cycle by two-site enzyme immunoassay. Clin Endocrinol (Oxf), 1994, 40: 717-723

162 Groome N P, Illingworth P J, O'Brien M, et al. Measurement of dimeric inhibin B throughout the human menstrual cycle. J Clin
Endocrinol Metab, 1996, 81: 1401-1405

163 Hall J E, Welt C K, Cramer D W. Inhibin A and inhibin B reflect ovarian function in assisted reproduction but are less useful at predicting outcome. Hum Reprod, 1999, 14: 409-415

164 Hartge P. Genetics of reproductive lifespan. Nat Genet, 2009, 41: 637-638

165 Kok H S, van Asselt K M, van der Schouw Y T, et al. Genetic studies to identify genes underlying menopausal age. Hum Reprod Update, 2005, 11: 483-493

Open Access This article is distributed under the terms of the Creative Commons Attribution License which permits any use, distribution, and reproduction in any medium, provided the original author(s) and source are credited. 